\title{
New Approaches for Detecting Groups of Targets
}

\author{
S.D. Halversen, G.J. Owirka, and L.M. Novak \\ MIT Lincoln Laboratory
}

\begin{abstract}
In military operations, targets are often deployed in well defined groups, such as tank divisions. It is possible to exploit this information and develop algorithms to detect such groups of targets for automated target cueing (ATC). These candidate areas would then be revisited by a higher resolution sensor. This paper describes one such ATC algorithm which significantly reduces the amount of area revisited by an automatic target recognition (ATR) system. In addition, we demonstrate that performance can be greatly improved by reconfirming the existence of a valid target group during the higher resolution revisits.
\end{abstract}

\section{Introduction}

In support of the DARPA-sponsored Critical Mobile Targets program, Lincoln Laboratory has developed a complete, end-to-end, automatic target recognition (ATR) system using high resolution $(0.3 \mathrm{~m} \times 0.3 \mathrm{~m})$, fully polarimetric, Ka-band SAR data; the performance of this system has been documented in [1]. Recently, there has been interest in the use of automatic target cueing (ATC) followed by automatic target recognition (ATR). In the ATC/ATR system scenario, data from a lower resolution (say $1 \mathrm{~m} \times 1 \mathrm{~m}$ ) sensor would be used to initially locate groups of targets in the SAR image; ATC algorithms and/or human interpreters would be used to select and prioritize detected target groups. The areas would then be revisited with a higher resolution sensor (say $0.3 \mathrm{~m} \times 0.3 \mathrm{~m}$ ) and multiple looks.

An example of a crude ATC group detection algorithm is as follows: by using a priori knowledge of the number of targets expected in a group - and detection locations from a prescreener - it would be possible to find those areas that contain the requisite number of targets. It is not unreasonable to assume the approximate number of targets in a group would be known. Often targets of interest are deployed in well defined groups, such as tank divisions.

This paper presents a more sophisticated group detection algorithm that uses results from the discrimination stage of our baseline ATR system developed for use with $1 \mathrm{~m} \times 1 \mathrm{~m}$ resolution data. Spatial groups are formed containing the objects with the best discrimination scores; then the discrimination scores of the individual objects within each group are averaged into a. single metric, which estimates how "target-like" the group is (group score). The group score can be used to prioritize the most "target-like" groups for ATR processing at higher resolution. The use of discrimination scores (instead of detection statistics) in a grouping algorithm is desirable because discrimination scores are estimates of how "target-like" the objects are within each group. As the results of our study show, the new group detection algorithm retains true target groups and rejects a large fraction of the false alarms.

The data used in this study consists of $231 \mathrm{~km}^{2}$ of 20 degree depression angle stripmap clutter from several areas in the northern United States; these data have varying amount of cultural content from severe to benign. Twenty-four target groups were contained within the data set; each target group contained 6 to 11 targets. Eight of the target groups consisted of camouflaged targets; in all other groups the targets were uncamouflaged. All of the data used for ATC were single polarization $(\mathrm{HH})$ with a resolution of $1 \mathrm{~m} \times 1 \mathrm{~m}$.

\section{Algorithm Details}

Figure 1 shows a block diagram of the ATC system consisting of three stages. Each stage will be described in the following subsections.

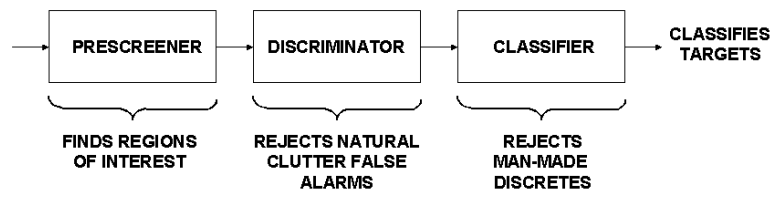

Figure 1: Block diagram of the $1 \mathrm{~m} \times 1 \mathrm{~m}$ resolution ATC system.

\section{CFAR}

In the first stage of processing, a two-parameter constant false alarm rate (CFAR) detector [2] is used as a prescreener; this stage of processing locates targets in the image on the basis of local radar contrast. Since a single target may produce multiple detections, the CFAR detections in target-size regions are clustered (grouped) together. Then a $30 \mathrm{~m} \times 30 \mathrm{~m}$ region of interest (ROI) around the centroid of each cluster is passed to the discrimination algorithm for further processing.

\section{Discrimination}

The second stage of processing, called discrimination takes as its input each ROI and analyzes it. The goal of discrimination processing is to reject clutter false alarms while accepting real targets. This stage consists of three steps: (1) estimating the size of the detected object, and eliminating those objects that are either too large or too small to be targets, (2) computing a set of discrimination features that measure the size, texture, and spatial properties of the detected objects, and (3) combining the features into a discrimination score that measures how "target-like" the detected object is. 
Morphological processing is used to estimate the size of the detected object. This consists of performing "dilations" and "erosions" with various kernels to form a morphological "blob" covering the detected object. The diameter of the blob is then calculated, and those objects which are grossly undersized or oversized, are eliminated.

In step two, discrimination features are calculated, which include: standard deviation, weighted rank fill, fractal dimension, normalized square rotational inertia, mean CFAR, and others $[3,4]$.

In step three of the discrimination stage a subset of these features (depending on polarization and resolution) are combined into a single discrimination statistic, the quadratic distance:

$$
\mathrm{d}_{\mathrm{t}}(\underline{\mathrm{X}})=\frac{1}{\mathrm{n}}(\underline{\mathrm{X}}-\underline{\hat{\mathrm{M}}})^{\prime} \hat{\Sigma}^{-1}(\underline{\mathrm{X}}-\underline{\hat{\mathrm{M}}})
$$

where $\mathrm{n}$ is the number of features, $\underline{\hat{\mathrm{M}}}$ and $\hat{\Sigma}$ : are estimates of the mean vector and covariance matrix of the features, and $\underline{X}$ is the vector of features measured from the object in question. The estimates for the mean vector and covariance matrix are calculated from an independent set of target data. The metric $\mathrm{d}_{\mathrm{t}}(\underline{\mathrm{X}})$ represents the distance of the detected object to the target class [5]. For an actual target, the quadratic distance calculated will be small, whereas most clutter false alarms will have a large quadratic distance and will be considered less "target-like."

\section{Grouping}

In the grouping stage, the spatial location of each object and its quadratic distance score are used. Only those objects with a small quadratic distance ("target-like") are used for spatial grouping. The grouping algorithm considers two objects to be in the same group if they are within 200 meters of each other. Additional objects will be considered to be part of the group if they are within 200 meters of any other object within the group.

Groups are eliminated if they contain less than 5 objects (too few to be a group of targets), or if they contain more than 30 objects (likely to be highly cultural areas, i.e. buildings). The average of the discrimination scores over the objects in the group is calculated and is considered to be the "group score." We then declare a group detection if the group score is below a certain threshold. That is, if, on average, the objects in the group are "target-like." Note that it is not necessary to detect all the targets in a deployment in order to detect the group of targets; it is only necessary to detect a minimum of 5 targets in the group.

As shown in Figure 1, the grouping algorithm was able to detect all 24 true target groups with 32 false groups detected in the $231 \mathrm{~km}^{2}$ of data. A typical false group is shown in Figure 2. If all groups are subsequently revisited by another sensor, the area re-imaged will be less than $5 \%$ of the original area.

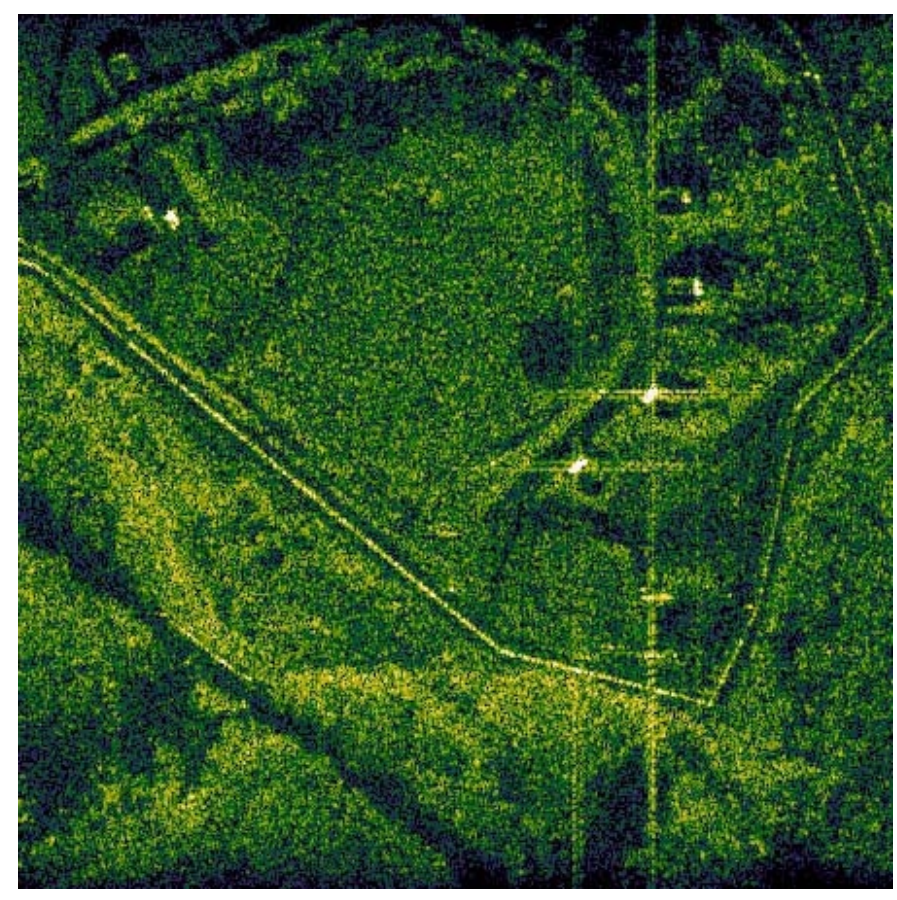

Figure 2: Typical false group.

\section{Revisitation}

In an ATC/ATR scenario these detected groups would be revisited with a high resolution sensor to collect data for automatic target recognition. However, we show that it is possible to dramatically reduce the number of false groups by simply reconfirming each group using the higher resolution data.

For each group detected using the $1 \mathrm{~m} \times 1 \mathrm{~m}$ resolution data, the corresponding area (in $0.3 \mathrm{~m} \times 0.3 \mathrm{~m}$ resolution) containing all of the objects in the group was processed through a modification of our ATR system developed for $1 \mathrm{~m} \times 1 \mathrm{~m}$ resolution data. A block diagram of this algorithm is shown in Figure 3. This algorithm is similar to that used for the lower resolution data, although it has the addition of a mean-squared error (MSE) classifier, described below, and an additional grouping stage. The reason for two grouping stages is for reduced computation. Typically, the classification algorithm is the most computationally intensive portion of an ATR system; reducing the amount of data processed through the classification algorithm significantly reduces the overall computation load. By reverifying the group status after discrimination, many false groups are eliminated. Hence, the advantage of a grouping stage after discrimination. 


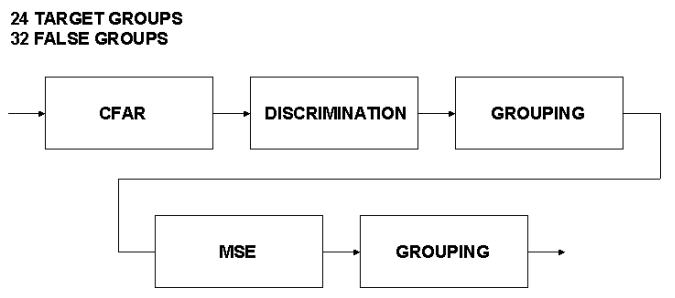

Figure 3: Block diagram of the $0.3 \mathrm{~m} \times 0.3 \mathrm{~m}$ resolution revisitation grouping.

\section{MSE Classification}

The mean-squared error (MSE) pattern-matching classifier is used to reject cultural false alarms caused by manmade clutter discretes and to classify the remaining target detections by vehicle type. In this study we implemented a two-class classifier (tactical target, and clutter) for the higher resolution data. The pattern-matching references used in the classifier were constructed by averaging five consecutive spotlight-mode images of a target collected at $1^{\circ}$ increments of azimuth, yielding 72 smoothed references for each type of tactical target.

To construct the reference templates for the MSE classifier, the pixel values (converted to $\mathrm{dB}$ ) of bare target training images are thresholded to eliminate all but the brightest and dimmest $5 \%$ of the data. This procedure reduces the original target image to a collection of target pixels and target shadow pixels. A binary image of the remaining pixels is then generated and morphologically processed to create a binary mask; all of the pixels in the original $(\mathrm{dB})$ image which are not under the mask are used to calculate a mean $\mathrm{dB}$ clutter level. The target image is then normalized by subtracting this clutter mean from the entire image. With this normalization scheme, the average clutter is removed, but the energy of the reference target relative to the clutter background is retained; therefore, the templates retain information about the power reflected from a target without relying upon the absolute calibration of the imagery. This makes targets and clutter more separable. After normalization, the target images are extracted using a rectangular mask that is just slightly larger than the target and is oriented at the same angle as the target. This eliminates nearly all of the clutter from the classifier templates.

When an ROI is passed to the classifier, the mean clutter level is calculated and the ROI is normalized (as described above). The mean-squared error between the ROI and each of the stored classifier templates in the reference library is calculated, and the template that minimizes the error is chosen as the best match. The reference template is translated a small amount in range and azimuth to compensate for any small errors in target centroiding, and the minimum error over these translations is taken as the best match for that template.
As with the discrimination stage, the lower the MSE score is, the more "target-like" the object in question is; thus it is possible to use the grouping algorithm with only minor changes

\section{Results}

The $0.3 \mathrm{~m} \times 0.3 \mathrm{~m}$ resolution revisitation emulation was performed (1) assuming we used a single polarization $(\mathrm{HH})$ sensor, and (2) for comparison, assuming we used a fully polarimetric sensor. For the fully polarimetric data, the $\mathrm{HH}$, $\mathrm{HV}$, and VV polarization channels are combined with a polarimetric whitening filter (PWF) to optimally reduce speckle [6]. Overall performance results are shown in Table 1. In each case, it was required that all true target groups be detected $\left(P_{d}=1.0\right)$, resulting the listed number of false alarm groups. As would be expected, using fully polarimetric (PWF) data reduces the number of false groups as compared to single polarization $(\mathrm{HH})$ data.

Table 1: Grouping performance results for revisitation using $0.3 \mathrm{~m} \times 0.3 \mathrm{~m}$ resolution sensor.

\begin{tabular}{|l|c|c|}
\cline { 2 - 3 } \multicolumn{1}{c|}{} & Target Groups & False Groups \\
\hline HH Discrimination & 24 & 16 \\
PWF Discrimination & 24 & 2 \\
\hline HH Classification & 24 & 7 \\
PWF Classification & 24 & 0 \\
\hline
\end{tabular}

By varying the threshold needed to declare a group, we have constructed receiver operating characteristic (ROCs) curves and show group detection performance after each of the grouping stages. Figure 4 shows the performance when using single polarization data exclusively. Note that the overall performance for group detection improves at each stage. This is due to the fact that each successive stage $(1 \mathrm{~m} \times 1 \mathrm{~m}$ resolution discrimination, $0.3 \mathrm{~m} \times 0.3 \mathrm{~m}$ resolution discrimination, and $0.3 \mathrm{~m} \times 0.3 \mathrm{~m}$ resolution MSE classification) provides a more accurate measure of how "target-like" an object is.

Figure 5 shows the performance of the group detection algorithm when using a fully polarimetric sensor for the $0.3 \mathrm{~m} \times 0.3 \mathrm{~m}$ resolution revisitation. By comparing Figures 4 and 5 it is clear that using fully polarimetric data for revisitation will provide about a factor of 5 reduction in the number of false groups after discrimination, and that after using the MSE classifier it is possible to get perfect performance from the group detection algorithm. 


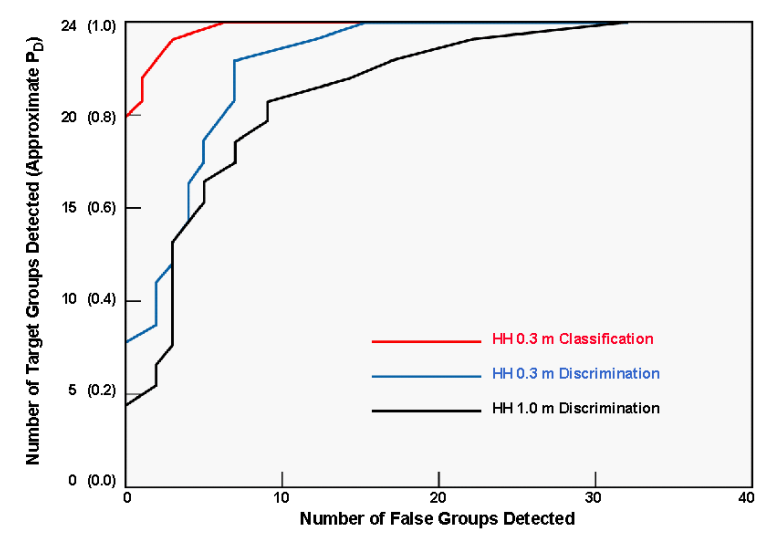

Figure 4: Grouping performance using $\mathrm{HH}$ data for $0.3 \mathrm{~m} \times 0.3 \mathrm{~m}$ revisitation.

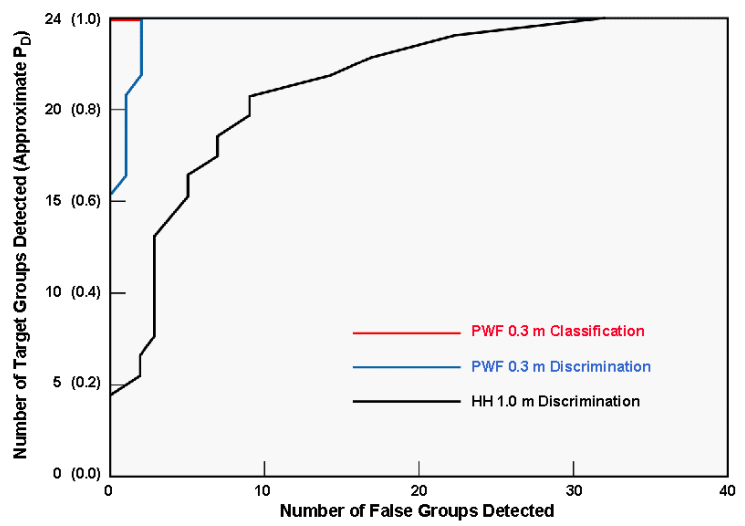

Figure 5: Grouping performance using PWF data for $0.3 \mathrm{~m} \times 0.3 \mathrm{~m}$ revisitation.

\section{Conclusions}

In this paper, we examined a detection algorithm designed for finding groups of targets, such as tank divisions, using $\mathrm{lm}$ $\mathrm{x} \mathrm{lm}$ resolution single polarization data. The assumptions used for target groups are relatively mild: each target group is assumed to have between 5 and 30 targets, and that the targets in a group are within $200 \mathrm{~m}$ of one another. By applying these assumptions, along with a measure of how "target-like" each detected object is, using $231 \mathrm{~km}^{2}$ of data it was possible to detect all of the true target groups contained within the data. In addition, 32 false groups were detected. While this seems like a high false alarm rate, it is important to note that the amount of area that would be revisited with a higher resolution sensor and/or examined by a SAR interpreter, would be reduced by at least a factor of 20 .
Those areas that were found to have candidate groups were re-examined using higher resolution $(0.3 \mathrm{~m} \times 0.3 \mathrm{~m})$ SAR data in an effort to reduce the number of false groups. This is considered to be the high resolution revisitation by another sensor. By attempting to reverify the existence of each group at the higher resolution it is possible to reduce the number of false groups significantly - to 7 false groups when using single polarization data, and rejecting all the false groups when using fully polarimetric data.

\section{References}

[1] L.M. Novak, et al, "Performance of a High Resolution Polarimetic SAR Automatic Target Recognition System," Lincoln Laboratory Journal, Vol. 6 No. 1, Spring 1993.

[2] G.B. Goldstein, "False Alarm Regulation in Log Normal and Weibull Clutter," IEEE Transactions AES, January 1973.

[3] M.C. Burl, et al, "Texture Discrimination in Synthetic Aperture Radar," 23rd Asilomar Conference on Signals, Systems, and Computers, Pacific Grove, CA, November 1989.

[4] D.E. Kreithen, et al, "Discriminating Targets from Clutter," Lincoln Laboratory Journal, Vol. 6 No. 1, Spring 1993.

[5] K. Fukunaga, et al, "The Acquisition Probability for a Minimum Distance One-Class Classifier," IEEE Transactions AES, July 1987.

[6] L.M. Novak, et al, "Optimal Processing of Polarimetric Synthetic-Aperture Radar Imagery," Lincoln Laboratory Journal, Vol. 3 No. 2, Summer 1990. 